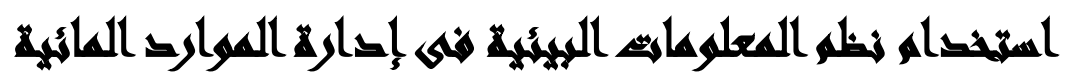

\section{هis}

$[r \leqslant]$

محمد فاروق أبو عارف(')- أحمد فؤاد مندور(r) - نهي سمير دنيا(')

كريم مصطفى جوهر (r)

() معهد الدراسات والبحوث البيئية، جامعة عين شمس ك) كلية التجارة، جامعة عين شمس (كس

\section{المستحلصى}

إن التحديات الحالية التى تواجه الموارد المائية المصرية قد أوجبت علينا دراسة أحدث

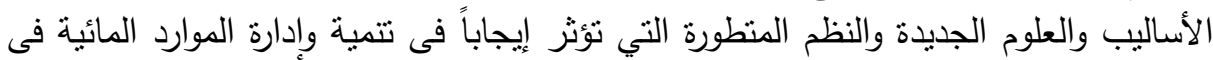

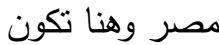

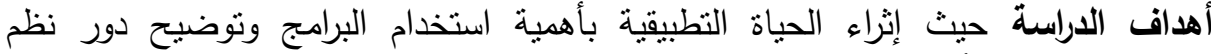

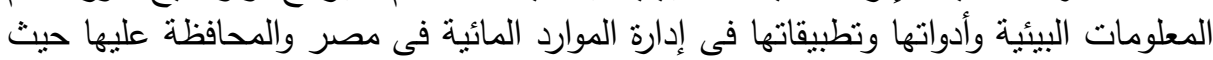

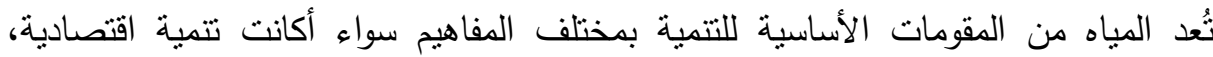

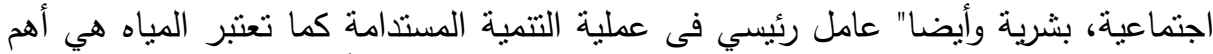

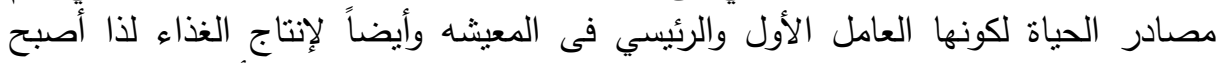

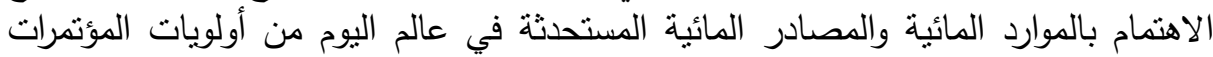

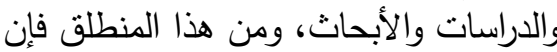

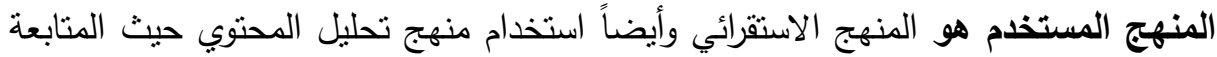

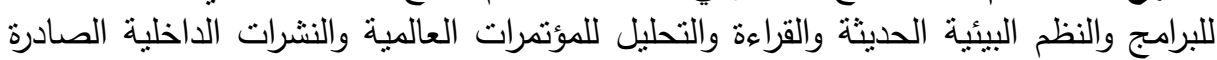

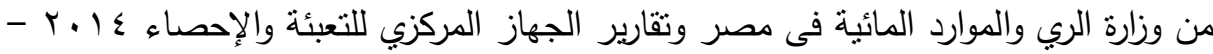

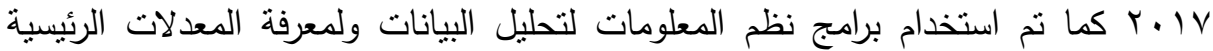

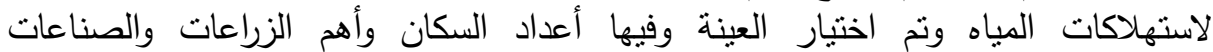

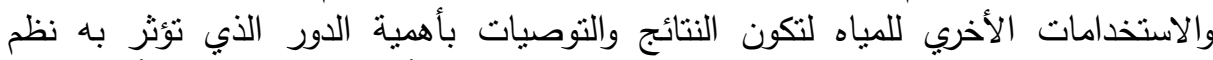

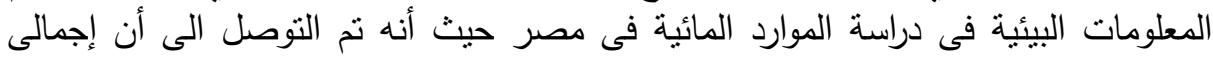

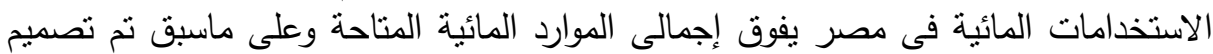

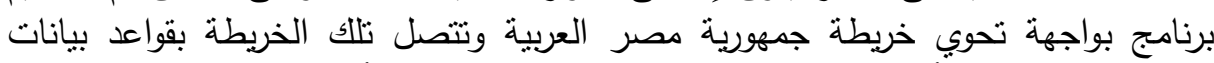

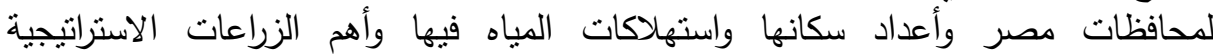

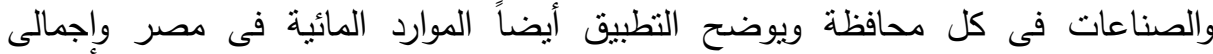
استخداماتها وهذا هو مايصبو إليه التطبيق فى تحقيق التتمية المستدامة للموارد المائية فى في النى 
الكلمات المفتاحية: نظم المعلومات البيئية، إدارة الموارد المائية، التتمية المستدامة

\section{Maradl}

البيئة هى أحد أهم الاتجاهات التى نالت اهتمام كبير خلال السنوات الأخيرة فى مختلف المجتمعات وذلك لدورها الأساسى فى الحياة وتعتبر المياه هى أهم مكون من مكونات البيئة

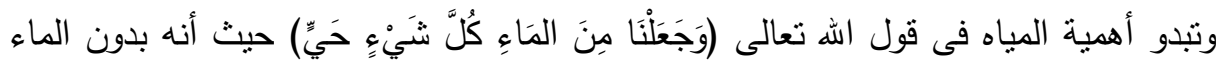
لا يتم تحقيق أى تقدم سواء كان زراعيا" أو صناعيا" أو إجتماعيا" كما تعتبر أيضا" أهم

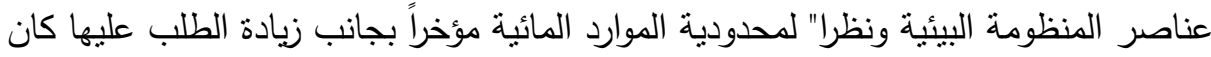

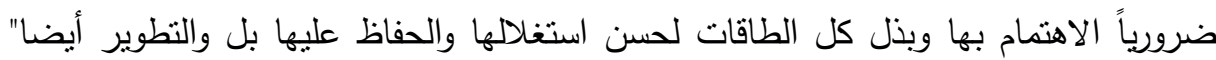

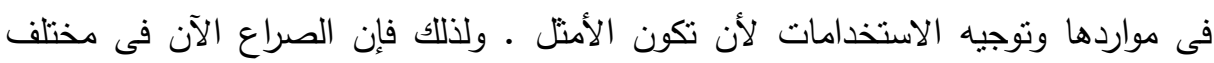

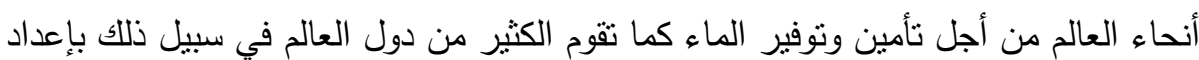
الخطط والسياسات, وتقديم الأبحاث والدراسات لتعظيم العائد من استخدامات الموارد المائية،

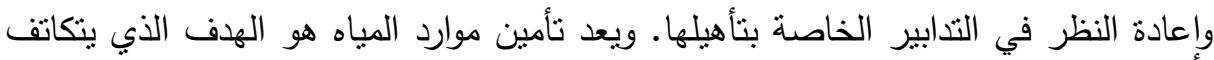

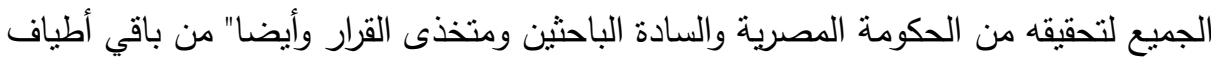

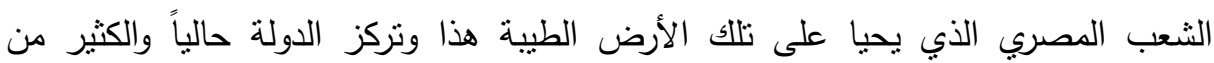

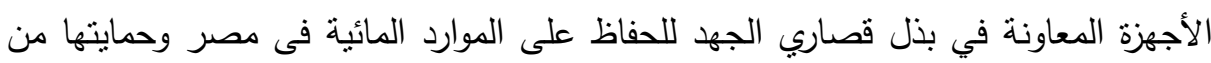
أشكال التلوث المختلفة حيث أن الكثير من الظروف المحيطة الحالية تؤدي بشكل كبير إلى لى تلوثها نتيجة للأنشطة المختلفة الصناعبة والزراعية والعمرانية والسياحية أيضاً، وتعتبر إدارة الموارد المائية في مصر قديمة قدم البلاد نفسها بداية" منذ الفراعنة وقياسهم لمستوى الفيضان

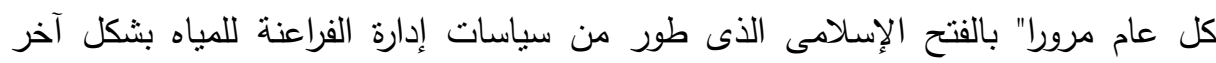
مختلف . وقد كان نابليون بونابرت أول من فكر في إدخال نظرية التخزين إلى الإدارة المائية تبعه فيها محمد علي باثا عندما أنثأ الترع الصيفية والقناطر الخيرية كوسائل للتخزين السطحي في النيل وفروعه حيث كان خزان أسوان أول محاولة علمية للتخزين خلال الفترة من

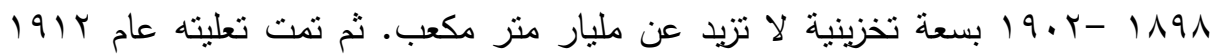




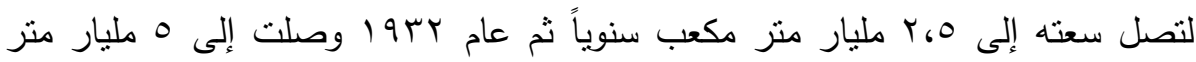

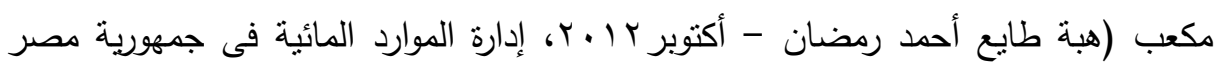
العربية، الإدارة العامة كلية الاقتصاد والعلوم السياسية جامعة القاهرة).

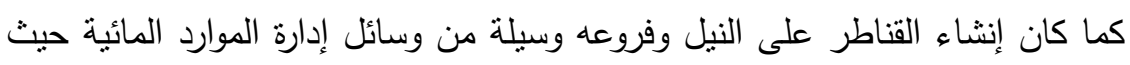

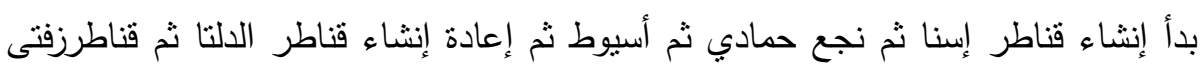
ودمياط على فرع دمياط وقناطر أدفينا على فرع رشيد.ثم تم إنشاء السد العالي في أوائل

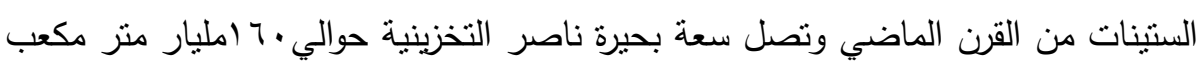

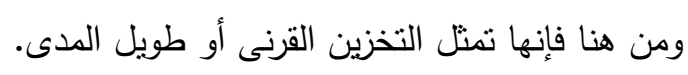
هذا وقد بدأت الإدارة العلمية للموارد المائية خلال عام

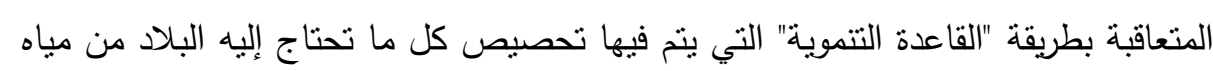

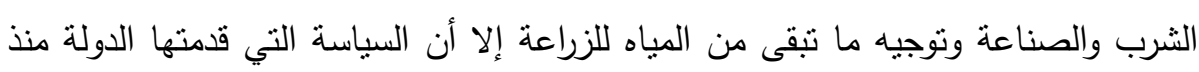

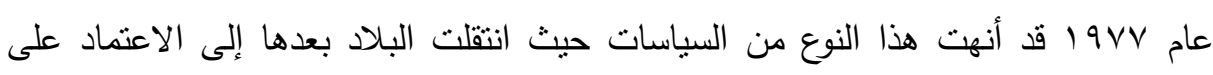

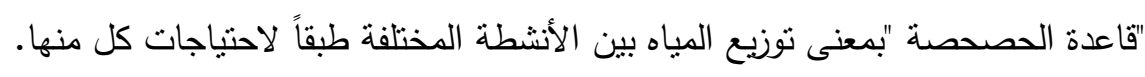
هنا يلزم التمييز بين نوعين من أنواع العرض للموارد المائية هما:

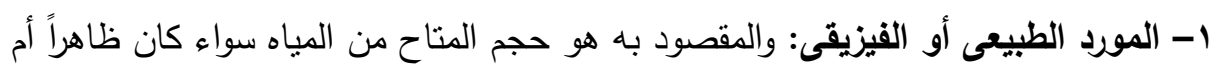
باطناً، مالحاً كان أو عذباً.

r - المورد المتاح: والمقصود به المورد المتاح فعليا للمياه من مختلف مصادر المياه، حيث أن هناك بعض المصادر التى من الصعب على الإنسان استغلالها اقتصادياً.

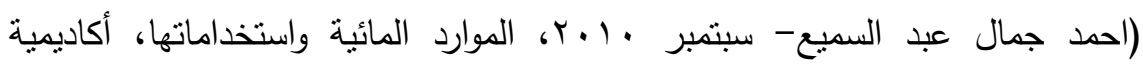
البحث العلمى، المركز المصرى الدولى للزراعة) ومما سبق يتضح أنه كان لابد لمصر نظراً لمحدودية الموارد المائية فيها وندرة الأمطار

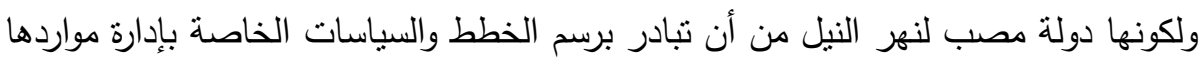
المائية ووسائل تتميتها لتغطي الاحتياجات المتزايدة في مجالات الاستخدام وعمل خطط لتنمية بردية

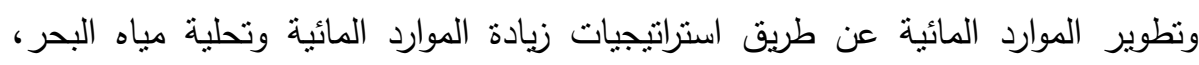

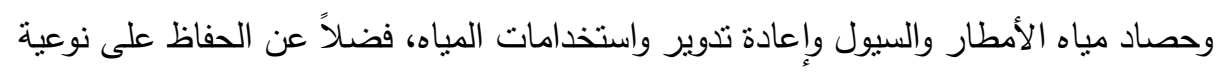


المياه.هذا وتواجه الإدارة المائية في مصر مشكلة على المستوى الداخلي ترجع إلى مجموعة

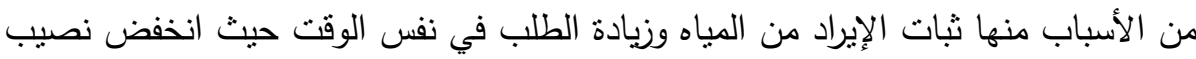
الفرد من الماء بينما تضاعفت احتياجات المواطنين من المياه نتيجة ارتفاع مستوى معيشتهر إضافة إلى استئثار الزراعة بالنصيب الأكبر من الميزانية المائية فضلاًعن مشكلة هدر المياه

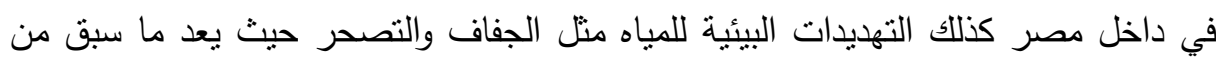
أهم أسباب المشكلة التي نواجه إدارة الموارد المائية في مصر • وعلى المستوى الخارجى حيث الكثير من التحديات الخارجية التى تتنافس للحصول على النصيب الكبير من الموارد المائية وعلى وجه الخصوص تللك الدول الششتركة فى الموارد المائية مع جمهورية مصر العربية.

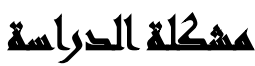

بعد أن تم الإطلاع على الدراسات السابقة ومنها دراسة رانيا صبرى عبد المنعم- بعنوان

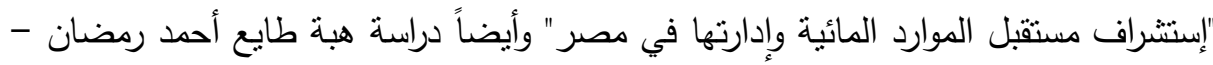
بعنوان "إدارة الموارد المائية فى جمهورية مصر العربية " واستقراء وتحليل التطورات والتغيرات فى مجال إدارة الموارد المائية في مصر ومراحلها بدءاً من الاهتمام بالمورد وتلبية الاحتياجات تنين أنه نوجد بعض المشكلات التي نواجه الموارد المائية فى مصر مثل سوء إداء الدارة الإيراد

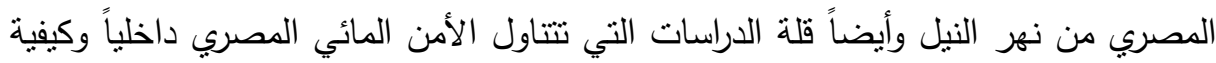

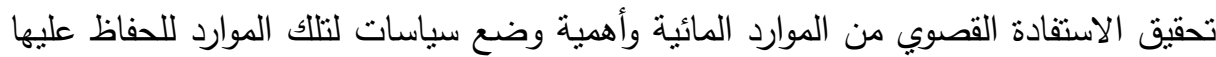

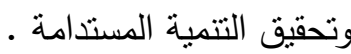

ومما لاشك فيه أن مصر حالياً تواجه تحديات كبيرة نتيجة لمواردها المائية المحدودة

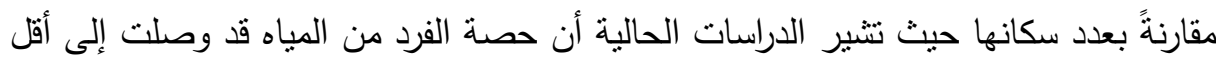
من . .0 مثر مكعب سنوياً، وهذا الرقم وفقاً للمعايير الدولية يشير إلى القرب من حد الفقر

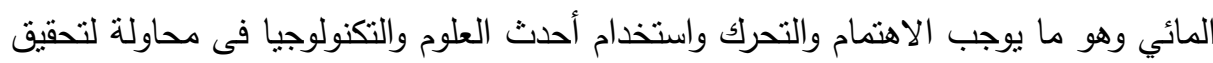
نتائج إيجابية والوصول إلى ما تصبو إليه الدولة من نتمية حديثة على مستويات متعددة 
إضافةً الى تلبية الحاجات الملحة لزيادة الإنتاج الغذائي وما يتطلبه ذلك من تكاتف الجهود فى

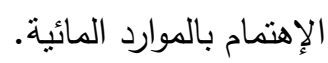

\section{أسئل التصاسم}

• إلى أى مدى تؤثر استخدامات المياه فى مصر بشكلها الحالى على تحقيق التتمية المستدامة؟ الماك

• ماهى أهداف نظم المعلومات البيئية فى إيجاد الحلول لتتمية الموارد المائية؟

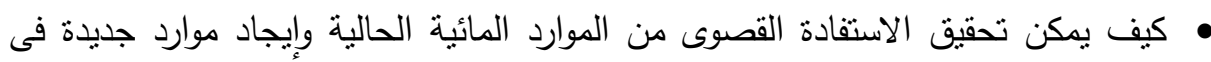

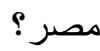
• ماهو الدور الذى تقوم به نظم المعلومات البيئية فى إدارة وتتمية الموارد المائية المصرية؟

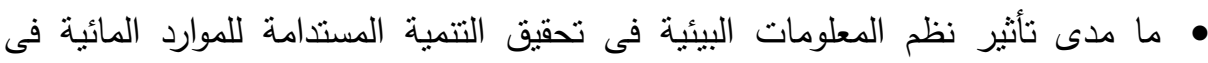
مصر؟

إن نظم المعلومات البيئية من أحدث العلوم التى تهتم بأفرع كبيرة وكثيرة فى العلوم البيئية

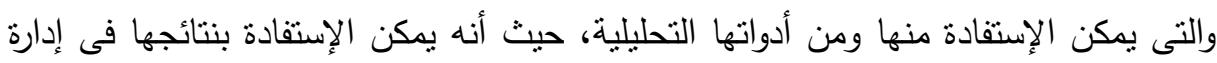
وتوزيع الموارد المائية المصرية المتاحة فى الوقت الحنه الحالى لتحقيق أفضل نتائج وتلبية

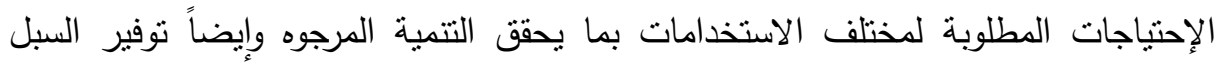
المختلفة لايجاد مصادر مائية مستحدثة والسعي لتحقيق التتمية المستدامة للأجيال القادمة.

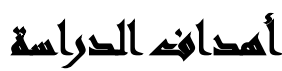

يسعي الباحثون من خلال تلك الدراسة إلى تحقيق الأهداف الآتية:

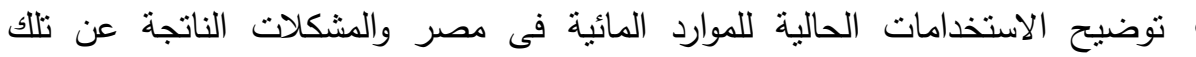
الاستخدامات ومدى خطورتها على تلك الموارد. • توضيح أهمية نظم المعلومات البيئية فى دراسة الموارد المائية المصرية.

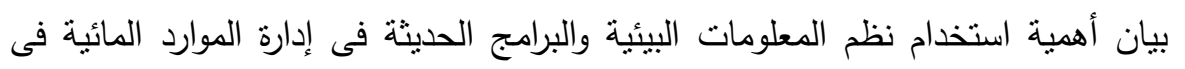

$$
\text { • متفيذ برنامج تطبيقي - نموذج لإختبار فروض الدراسة. }
$$




\section{الهمية التراسما}

ترجع أهمية تلك الدراسة البحثية لما يتوقع أن تحققه بتوفيق من رب العالمين وبالاستفادة من السادة الأساتذة وذللك على المستوبين العلمي والعملي على النحو النالي: 1 - على المستوى العلمى: حيث أن الأهية العلمية تتمثل في محاولة تحقيق مايلي: • توضيح إطار متكامل لقياس أهمية إدارة الموارد المائية فى مصر • • بلورة حقيقة أهمية دراسة نظم المعلومات البيئية وعلاقتها بالتتمية المستدامة وإدارة الموارد المائية. توفير دراسة لتوضيح العائد من تطبيق نظم المعلومات البيئية فى تتمبة الموارد

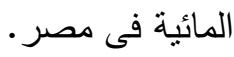

r - على المستوى العملى: يري الباحث أن الأهمية العملية تتمثل في محاولة تحقيق مايلي: تقديم دراسة وتطبيقاتها عن البيانات الكمية والبيانات النوعية وتوضيح فائدة تجميع وتخزين تلك البيانات للمساعدة فى توجيه تللك البيانات وعمل التحليلات البيانية والاستفادة بها فى نواحى إدارة الموارد المائية فى مصر .

\section{هزوضر التوباسلا}

تماثياً مع أهداف الاراسة الحالية فقد تم صياغة الفروض للاراسة كالأتى: • يوجد تأثير ذو دلالة معنوية لاستخدام نظم المعلومات البيئية فى إدارة الموارد المائية

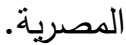
• يوجد تأثثر ملحوظ لاستخدام نظم المعلومات البيئية وأداواتها فى تحقيق التتمية المستدامة للموارد المائية فى جمهورية مصر العربية. 


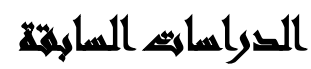

ا - دراسة: رانيا صبرى عبد المنعم(؟ . ץ) بعنوان: "استشراف مستقبل الموارد المائية وإدارتها في مصر"

مركز الدراسات المستقبلية التابع لمركز المعلومات ودعم إتخاذ القرار بمجلس الوزراء وفي تلك الدراسة تم تقديم العديد من المقترحات لمساعدة صانعي القرار في اتخاذ قراراتهم وفقاً للخيارات المتاحة فيما يتعلق بإدارة موارد المياه، وذللك من أجل توضيح حالة الموارد المائية

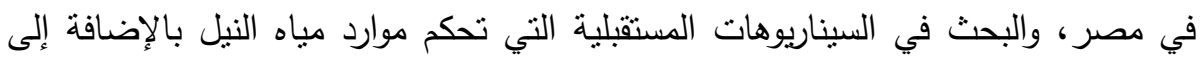
تحديد أهم القوى الدافعة التي تؤثنر على مستقبل الموارد المائية في مصر .

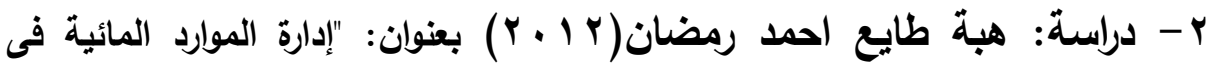
جمهورية مصر العربية - دراسة تقويمية"، الإدارة العامة من كلية الاقتصاد والعلوم السياسية، جامعة القاهرة

وفي هذه الدراسة تم تقديم عمل أكاديمي عن مشكلة واجهها المجتمع المصري وهي سوء إدارة

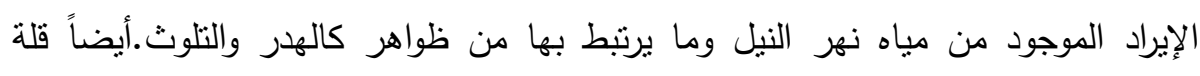

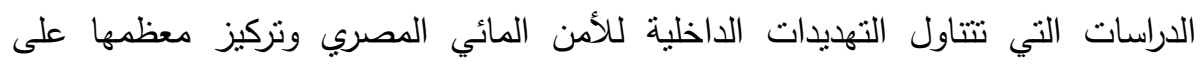
المخاطر الخارجية الموجودة والمحتملة من جانب دول حوض النيل، حيث تحاول الدراسة

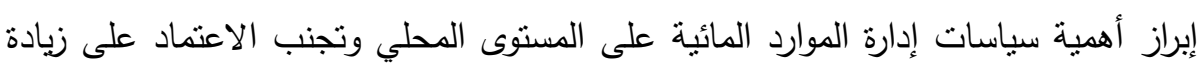

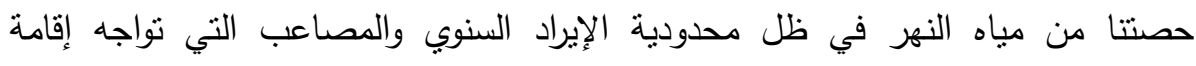
المشروعات المائية المصرية المقترحة في هذا الثنأن بالنظر للمشاكل السياسية التي تواجه

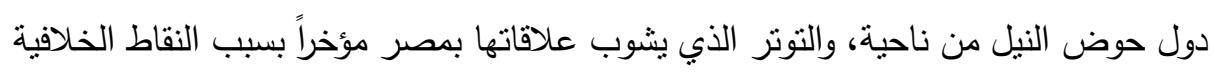
المعلقة في مبادرة حوض النيل من ناحية أخرى. 


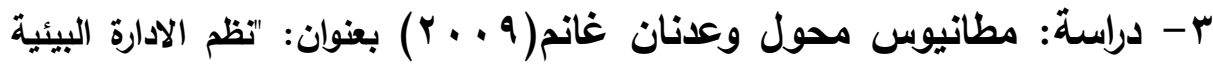
ودورها فى التنمية المستدامة"، قسم الاقتصاد - جامعة دمشق مجلة جامعة دمشق للعلوم

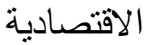

توصلت هذة الاراسة إلى أن البساطة فى أدوات تتفيذ نظم الإدارة البيئية وحداثتها وعلاقتها بالتتمية المستدامة يهدف إلى توسيع قاعدة التأهيل والتدريب فى مجال حماية البيئة التهات وقد جاءت هذه الدراسة لتوضح واقع نأثير نظم الإدارة البيئية ودورها فى التتمية المستدامة بسبب عدم اقتصار الششكلة البيئية على مكان محدد بل تخطته لتصبح من المشكلات العالمية

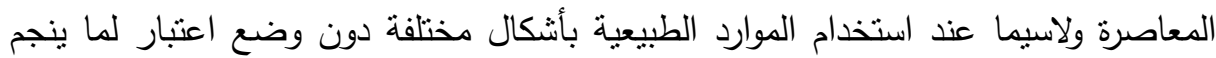
عن هذا الفعل والاستخدام ومدى تاثيرة على التوازن البيئى . ع - دراسة: حنان سعد الدين( . . . . ب) بعنوان: "بعض العوامل المؤثرة على اتجاهات

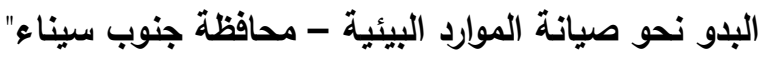
حيث استهدفت الاراسة التعرف على مستوي صيانة الموارد البيئية وتحديد أهم المتغيرات

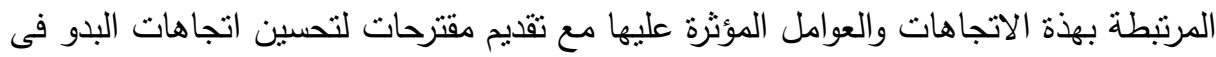
محافظة جنوب سيناء ( على سبيل المثال ) نحو صيانة الموارد البيئية - وخصوصاً الموارد

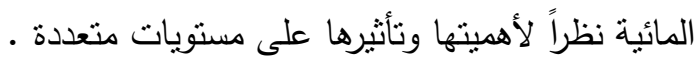
تنتمي تلك الدراسة الى الدراسات الوصفية التحليلية حيث استخدم فيها المنهج العلمي من خلال المسح الاجتماعي للعينة وذلك للكثف عن طبيعة العلاقات والاتجاهات نحو للتهات

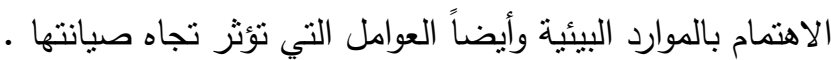

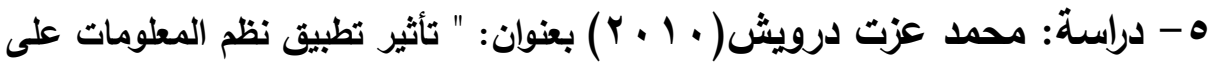

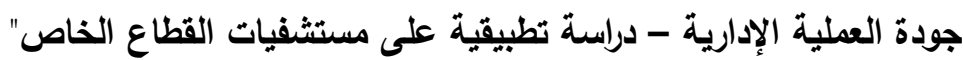
تهاف تلك الدراسة الى التعرف على المشكلات التي تواجه نظم المعلومات فى جودة العملية الادارية وتحديد اتجاهات الجودة في نظم المعلومات حيث يتضح من عمليات نظم

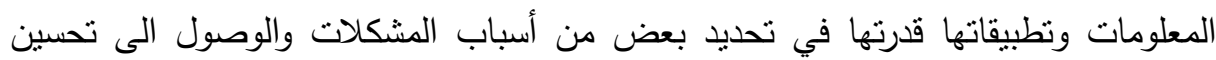
الأداء داخل إحدي المستشفيات كنموذج للنطبيق. 
وقت أثبتت الدراسة أنه يوجد نأثثر كبير وذلك فى نطبيق نظم المعلومات على جودة العملية الإدارية.

University of بعنوان Sustainability in Water Resources Management -California Department of Civil and Environmental Engineering اهتمت الدراسة باستعراض التطورات والتغيرات في مجال إدارة الموارد المائية، حيث

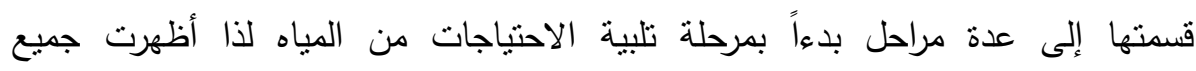

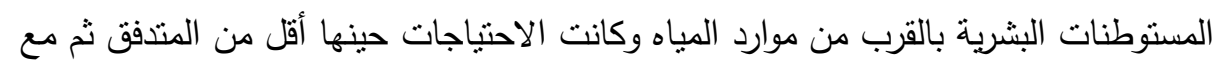

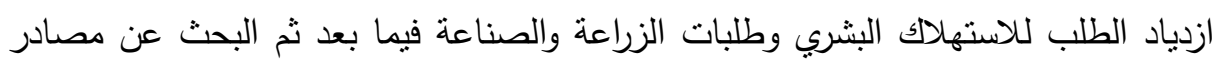

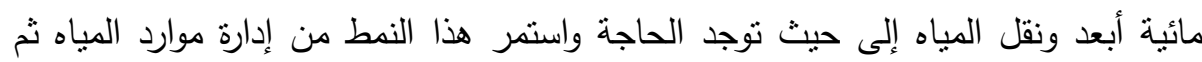

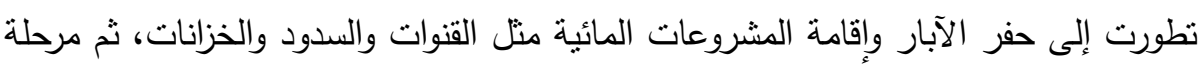
قضايا جودة المياه التي ظهرت مع استمرار تتامي الطلب على المياه وأيضا نطرقت الدراسة

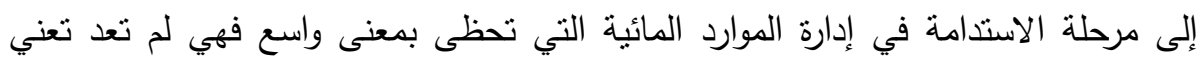

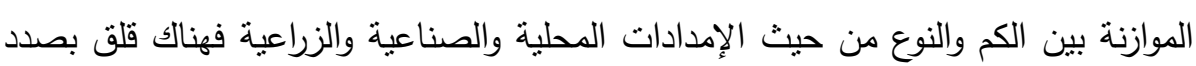
البيئة المائية والآثار السلبية على الموارد المائية وتحقيق الاستدامة.

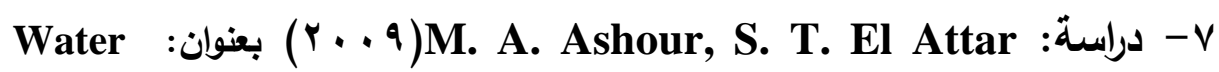
Resource Management in Egypt - Assiut University

أوضحت هذه الاراسة أن نظام الموارد المائية فى مصر يتكون من العديد من المكونات

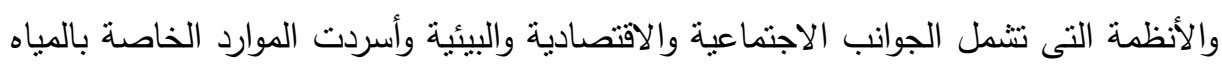

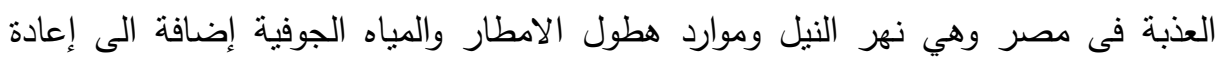

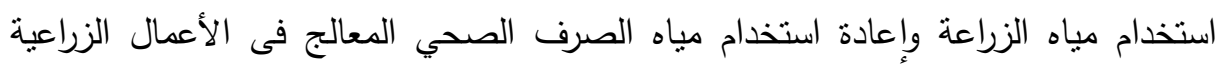
وأيضاً المصادر المستحدثة وأوضحت الدراسة أنه قد أنخفضت حصة الفرد من المياه سنوياً

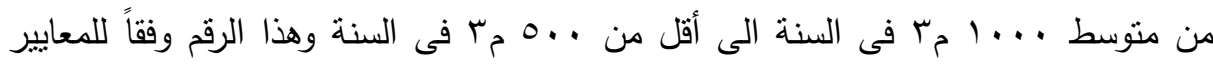

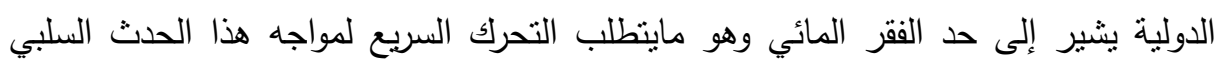
وإيجاد استراتيجيات للتعامل مع هذا الأمر . لـن 


\section{الإسار المعربهي للسوراسة}

تناولت الاراسة عدة مفاهيم منها:

نظم المعلومات البيئية: الذي يعني دمج وترتيب البيانات البيئية المفردة واجراء عمليات المعالجة للحصول على المعلومات المبوبة التي نساهم وتساعد فى تقديم الحلول البيئية التي لتي تحقق أهدافاً وتكون فى أولوياتها تحقيق التتمية المستدامه - ملخص من نشرة جمعية الأمم

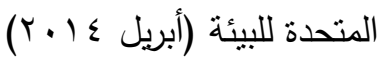
والموارد المائية: وهي تعتبر الأصول المائية الطبيعية التي يمكن استخدامها مباشرة أو بعد معالجتها للإنتاج أو للاستهلاك وتتقسم إلى موارد متجددة وموارد غير متجددة متل الأنهار

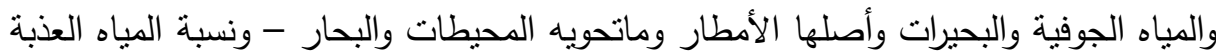
على سطح الكرة الأرضية لاتتعدي r\% بينما تغطي المياه المالحة (مياه المحيطات والبحار) النسبة العظمي منها والتي تقدر بحوالي 91\% أحمد فؤاد الخولي مجلة علوم المياه (أبريل

مفهوم إدارة الموارد المائية: وهي تعني إدارة العرض والطلب حيث أن إدارة العرض وتثمل كافة الأنشطة اللازمة لتحديد مواقع الموارد والمصادر الجديدة وتتميتها واستغلالها الاستغلال الأمثل وإدارة الطلب وهي نتشمل كافة الآليات اللازمة لتحقيق المستويات والأنماط

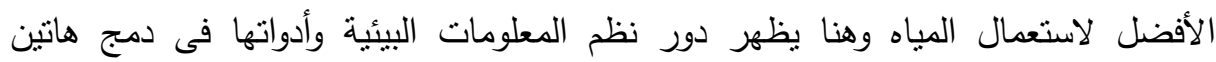
الإدارتين فى عملية واحدة للوصول إلى اختيار البدائل من خلال النطبيق محل الدراسة

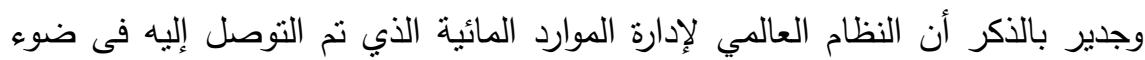
العديد من المناقثات الدولية حول كيفية التغلب على أوجه الضعف فى إدارة الموارد المائية

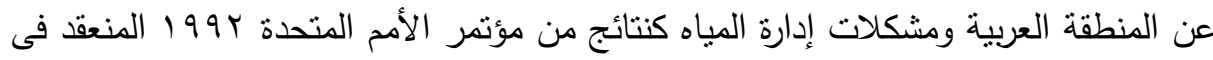
ريودي جانيرو يقوم على التحول عن النماذج التي كانت تركز على تتمية المصادر الجديدة للمياه من جانب العرض والتركيز على جانب الطلب والتدابير التنظيمية والسياسات التي ترمي لتري إلى التغلب على أوجه إخفاق الأسواق والحكومات وأيضاً إلى تنبي استراتيجيات قومية للمياه 
تكون إنعكاساً للأهداف الاجتماعية والاقتصادية والبيئية إضافةً إلى تدعيم أعمال البحوث والاهتمام بالعلوم الحديثة والتكنولوجيا لتحقيق إدارة حديثة للموارد المائية .

\section{منهمج القوراسة}

يجمع منهج الاراسة فى البحث بين عدة مناهج بحثية وذلك لخدمة الجانبين النظري والعملي:

فيما يتعلق بالجانب النظري: سيتم اتباع المناهج الدراسية البحثية التالية ذكرها وذلك كل

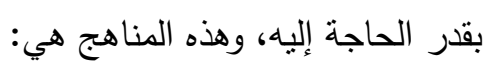

أولا" المنهج الاستقرائي: وفيه يتم استقراء البحوث والدهاء والداسات والرسائل العلمبه والدوريات

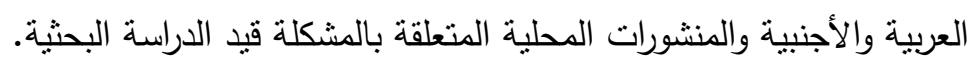
ثانيا" منهج تحليل المحتوي: ويتم فيه تحليل الأفكار والعلاقات والدذخل والجوانب العلمية والعملية التي تتنمل عليها البحوث والدراسات التي سيتم استقرائها بما يخدم الأهداف الموضوعه للاراسة البحثية.

ثالثاً المنهج الاستتباطي: وذلك بهدف استتباط أركان ومقومات قياس الأداء علي نتمية

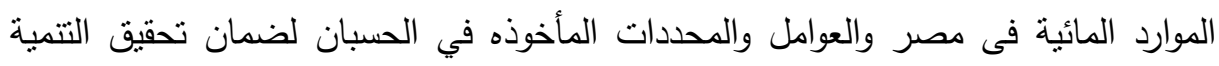
المستدامة باستخدام أحدث العلوم فى مجالات نظم المعلومات البيئية

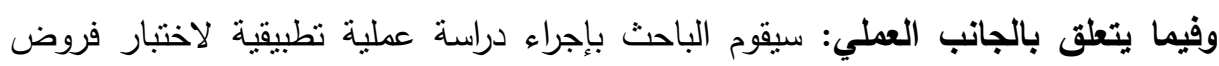

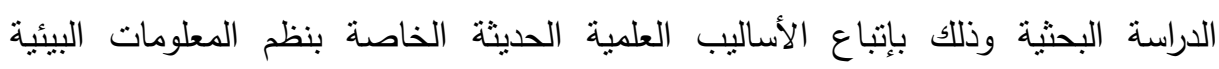

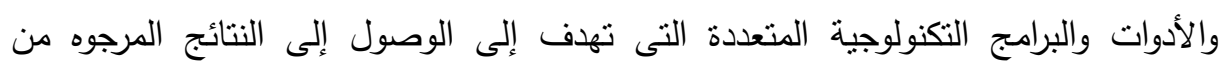

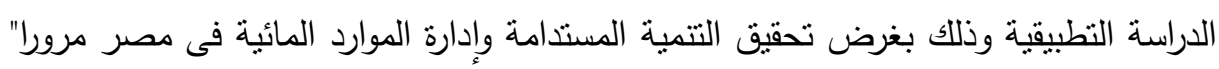

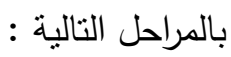

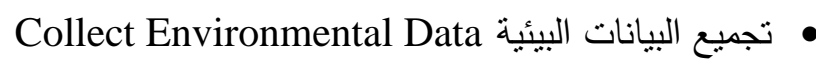

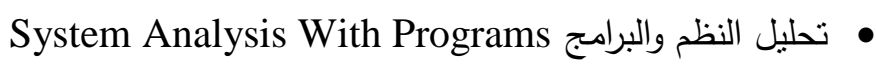

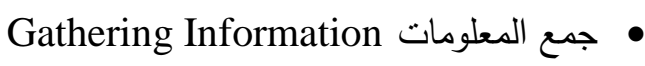

Store Information In C. Database تخزين المعلومات في قاعدة بيانات مركزية •

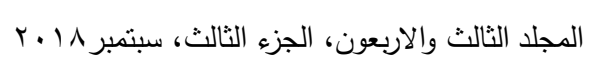


مجلة العلوم البيئية

معهز الدراسات والبحوث البيئية - جامعة عين شمس لهن

Participation for Improvement and المشاركة للتحسين والتطوير Development

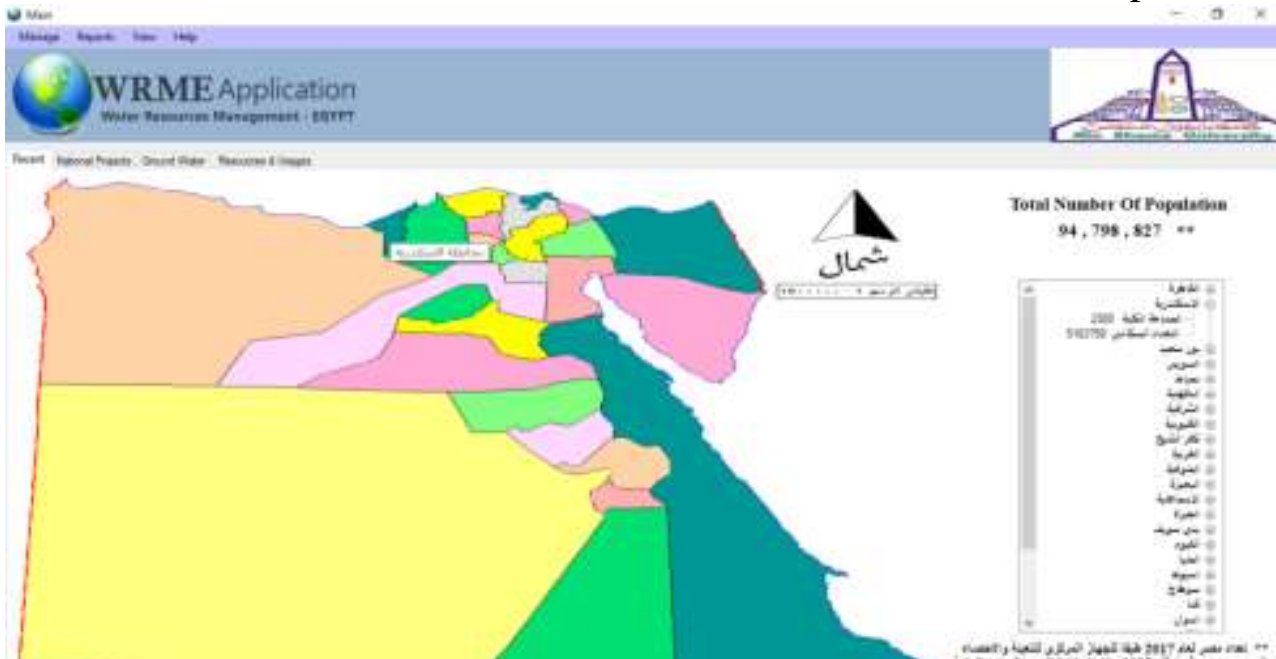

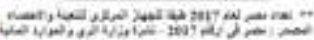
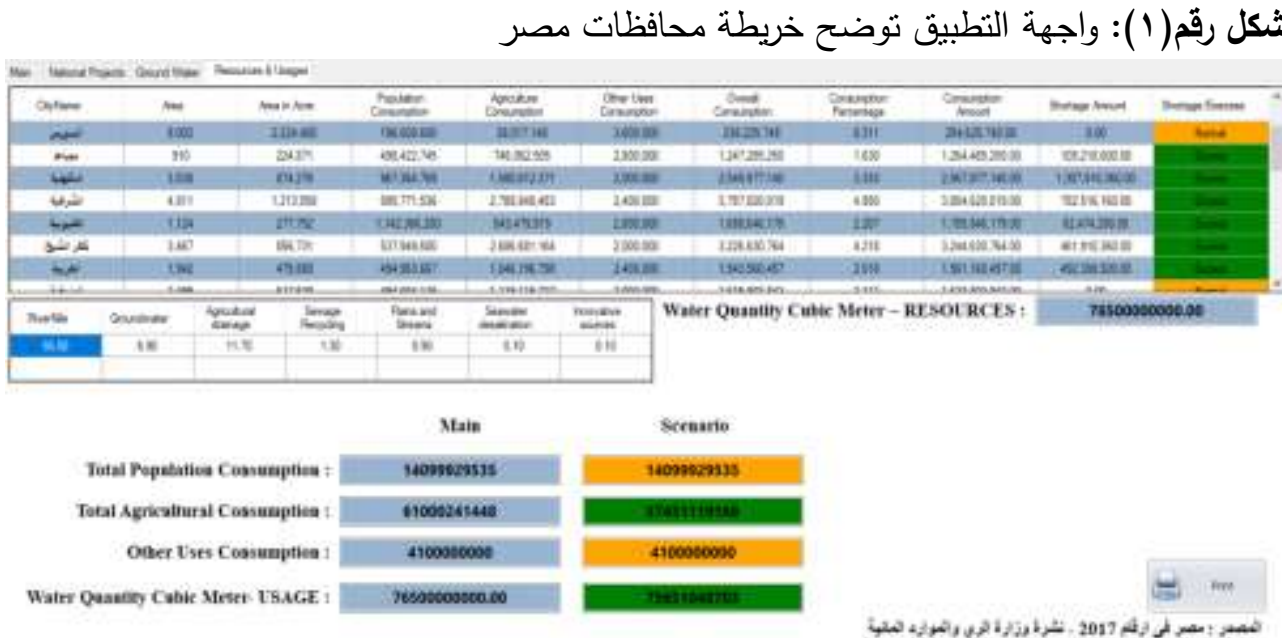

شكل رقم(ץ) : نموذج لمخرجات قاعدة البيانات من التطبيق

وحيث أن الهدف من الدراسة يتعلق بالموارد المائية فى مصر فقد نمات نم تجميع البيانات

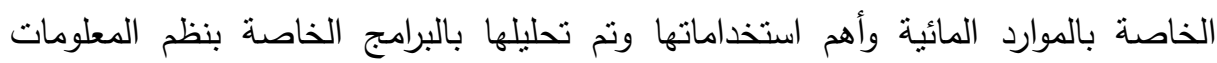


بمجالاتها المختلفة ودراساتها للوقوف على مدخلاتها ومخرجاتها وطرق معالجاتها وأهم نتائجها ومن ثم تم تجميع معلومات البحث من بعض المصادر منل جهاز شئون البيئة -وزارة الموارد المائية والرى - الجهاز المركزى للتعبئة والإحصاء وبعدها تم عمل بعض بعض من عمليات التحليل

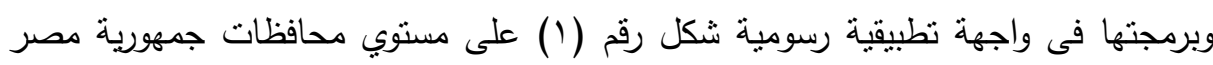
العربية وترتيباً لتنلك البيانات وسهولة العمل عليها تظهر كما فى قاعدة بيانات مركزية شكل

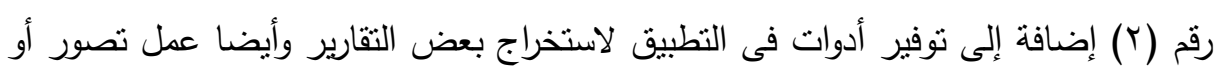

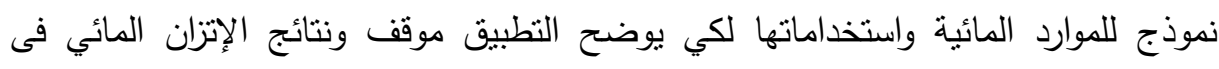

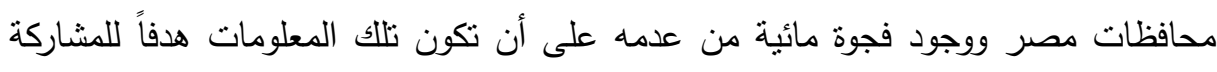

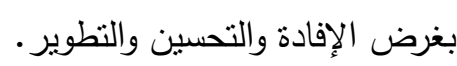

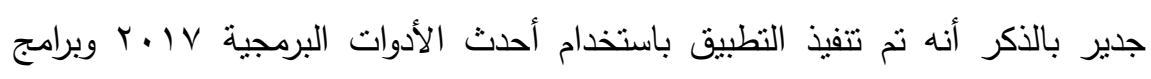

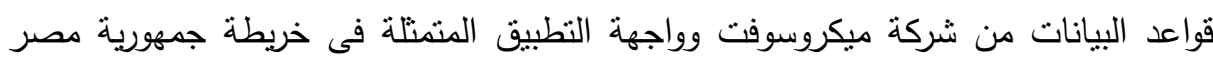

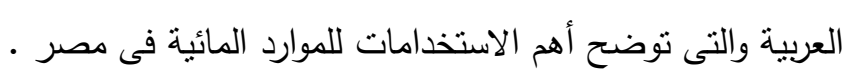
وحيث أن المنهج الاستقرائي هو أحد المناهج المستخدمة فى البحث فقد تم إختيار العينة

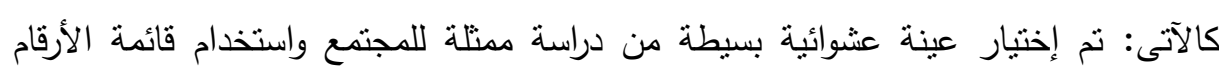

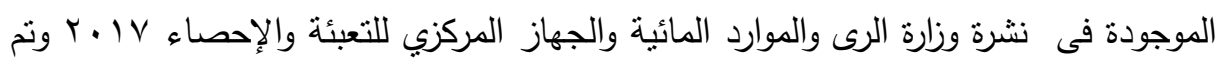

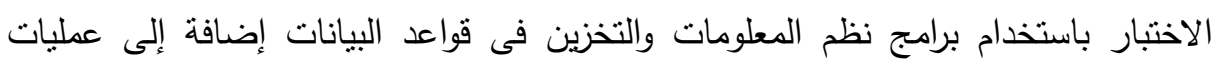

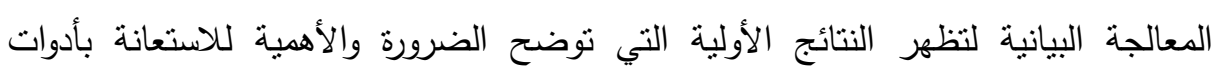
تطبيقية إضافية فى إدارة الموارد المائية فى مصر > لالجية

\section{اجزاءايه التراسة}

وتوضيحاً للاراسة الني أجراها الباحثون ودور التطبيق محل الدراسة فى إدارة الموارد

$$
\text { المائية فى مصر تمت الخطوات كالآتى: }
$$

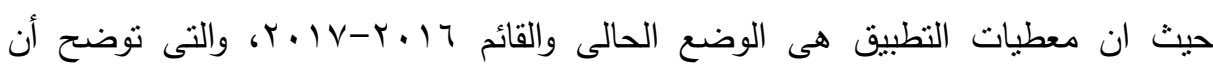

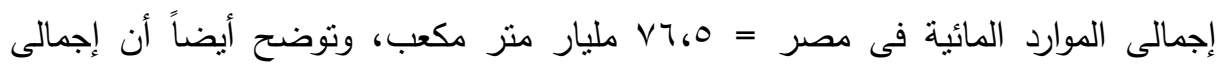

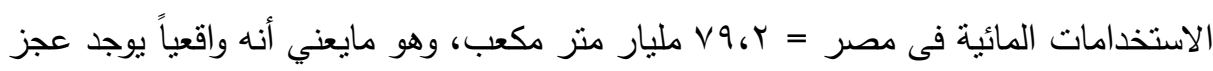

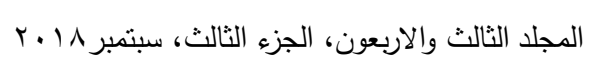


يُقددر بحوالى ثلاثة مليارات متر مكعب من المياه سنوياً وهو الفرق بين مجموع الموارد المائية

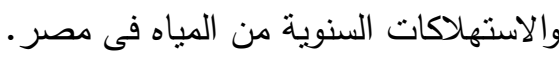

ولاستنضاح الدراسة وعند تتفيذ وعرض السيناريو المعتمد فى القرار الوزاري بجريدة

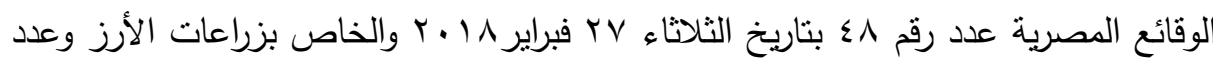
الأفدنة الخاصة بزراعة الأرز فى مصر داخل معطيات التطبيق محل البحث تظهر لنا لنا النتائج

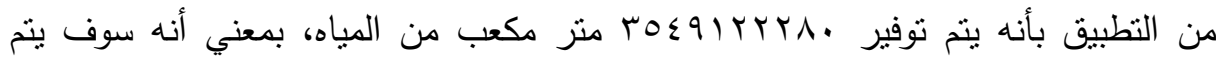

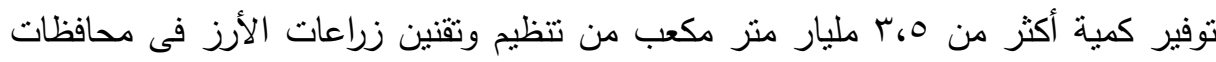
مصر وأيضاً تحقيق المحافظة على البيئة والتوازن البيئي خاصة فئ شئ شمال الدلتا والساحل الثمالى كما هو واضح فى نتائج قاعدة بيانات مركزية شكل رقم (Y). وهذا يبين دور البرنامج أو النطبيق محل الدراسة فى وضع وتقديبم النماذج والسيناريوهات المقترحة أو المتوقعة وتحليلها والاستفادة بنتائجها حيث يمكننا فى التطبيق اختبار نتائج نموذج وصنج على سبيل المثال فى الزراعة أو فى استخدامات المياه واستهلاكاتها بشكل عام ليقوم التطبيق بإظهار البيانات اللازمة وتوضيح النتائج التي توضح صنئي صحة النموذج من عدمه أو تحقيقه للهدف الرئيسي فى نوفير كميات من موارد المياه فى مصر من عدم التحقيق مما ينعكس

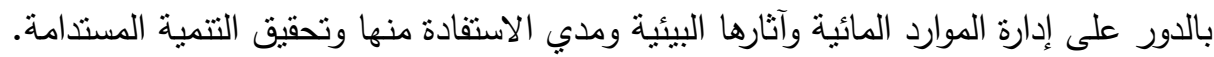

\section{المتخائي والموكيانت}

• ضرورة الدراسة والمتابعة المستمرة لبرامج وأدوات نظم المعلومات المتعددة للاستفادة منها

فى المجالات المختلفة وزيادة مصادر المعلومات فى الموارد المائية. • استخدام التطبيق المقترح وذلك لبيان أهمية نظم المعلومات فى إدارة الموارد المائية والاستفادة بنتائج التطبيق فى مصر بما يحقق الفائدة والتتمية المستدامة.

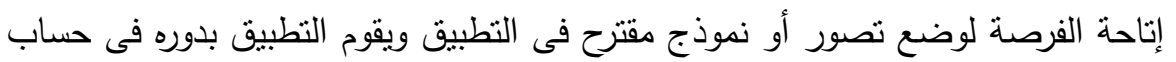
الاكثفاء أو وجود فجوة بين الموارد المائية واستخداماتها المختلفة.

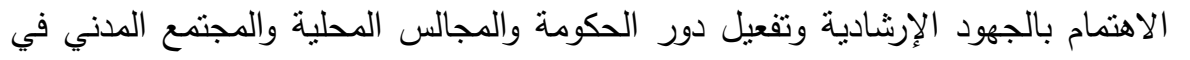
نشر الوعي البيئي وتطبيق الاستراتيجيات المنلي لإدارة واستخدام الموارد المائية في مصر . 600

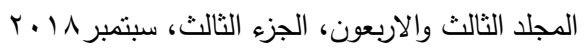




\section{sall}

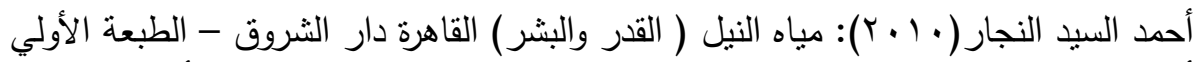

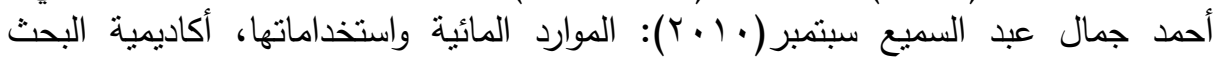

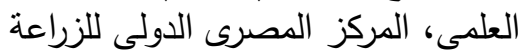

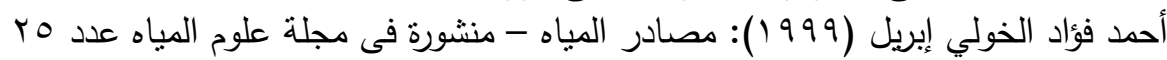

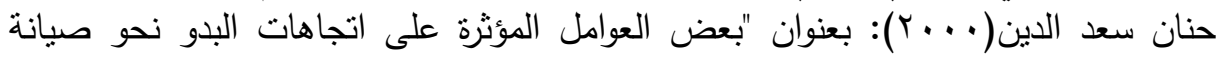

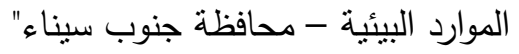

خيرى حامد العشماوى: " تقدير القيمة الاقتصادية لمياه الرى فى الزراعة الزية المصرية ،مجلة

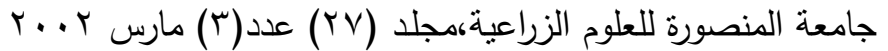

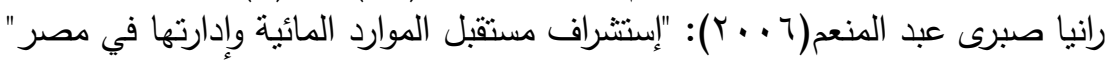

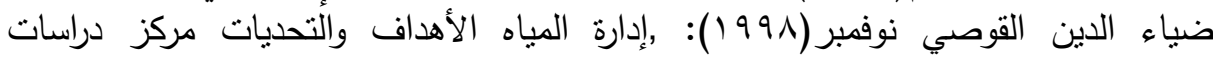

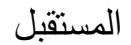

عبد الحكيم محمود(Y ( †): الطاقة المتجدة فى الوطن العربى، منظمة المجتمع العلمى

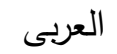

النشرة الإخبارية للمركز القومى للبحوث - منتدى بحوث المياه (تصدر ربع سنوية )

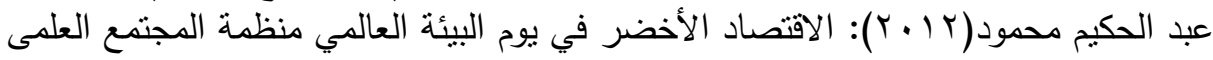

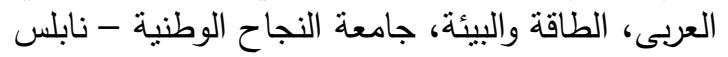

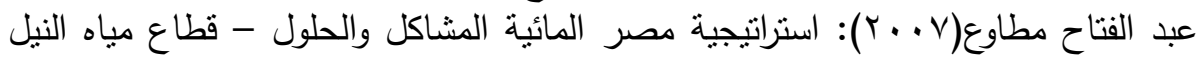
الموارد المائية وترشيد استخدامها في مصر . صدر عن الجهاز المركزي للتعبئة

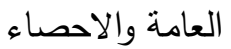

محمد عبد الهادى راضى، الإمكانيات المائية لمصر والتخطيط الأمتل لتتميتها واستخدامها

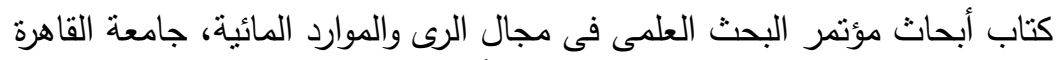

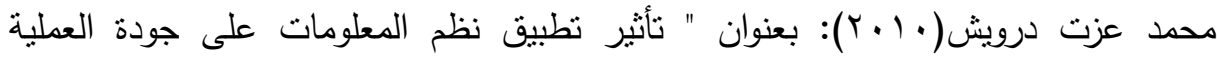

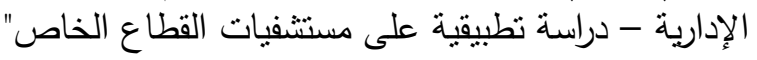

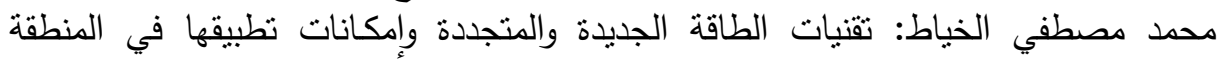

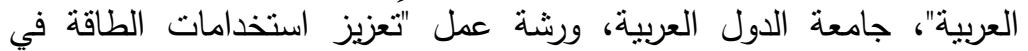

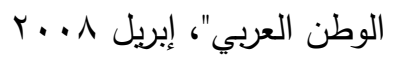

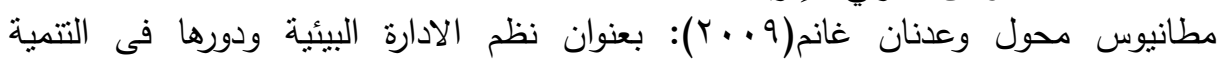

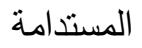

نجاة النيش(يونيو ( . ب): "التتمية المستدامة آفاق ومستجدات المعهد العربي للتخطيط الكويت

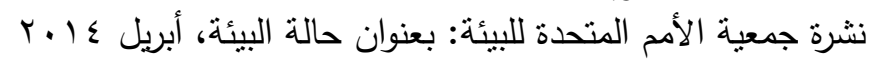




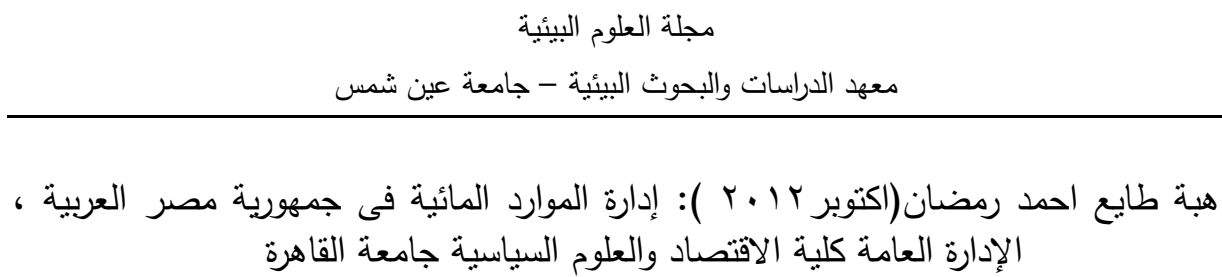

M. A. Ashour, S. T. El Attar (2009): Water Resource Management In EGYPT - Assuit University

Randa El Bedawy (2014): Water Resources Management: Alarming Crisis for Egypt - Journal of Management and Sustainability

Slawomir W. Hermanowicz (2014): Sustainability in Water Resources Management - University of California

\title{
USING OF ENVIRONMENTAL INFORMATION SYSTEMS IN THE MANAGEMENT OF WATER RESOURCES IN EGYPT
}

\author{
Abo-Aref, M. F. ${ }^{(1)}$; Mandour, A. F. ${ }^{(2)}$; Donia, Noha, . $^{(1)}$
} and Gohar, K. M. ${ }^{(2)}$

1) Institute of Environmental Studies and Research, Ain Shams University 2) Faculty of Commerce, Ain Shams University

\begin{abstract}
The current challenges facing Egyptian water resources have led us to study the latest methods, new sciences and advanced systems that positively affect the development and management of water resources in Egypt, The objectives of the study are to enrich the applied life with the importance of using the programs and to clarify the role of environmental information systems and their tools and applications in the management and conservation of water resources in Egypt. Water is one of the basic components of development in various concepts, whether it is economic, social and human development. It is also a major factor in the process of sustainable development. Water is also the most important source of life because it is the primary factor in
\end{abstract}

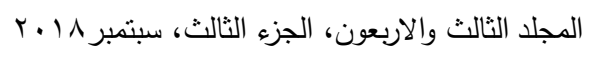


living and food production. Therefore, attention to water resources and water resources developed in today's world of priorities of conferences and studies and research, From this point on The methodology used is the inductive method and also the use of the content analysis approach, where follow-up programs and modern ecosystems, reading and analysis of global conferences and internal publications issued by the Ministry of Irrigation and Water Resources in Egypt and the Central Agency for Mobilization and Statistics 2014-2017 the Data systems programs were used to analyze the data and to find out the main rates of water consumption. The sample was selected in which the population numbers, the most important crops, industries and other uses of water To be the results and recommendations of the importance of the role that affects

the environmental information systems in the study of water resources in Egypt As it was concluded that the total water use in Egypt exceeds the total available water resources The program is designed with a map containing the Arab Republic of Egypt This map is related to the databases of the governorates of Egypt, its population, its water consumption, the most important strategic crops and the industries in each governorate. The application also shows Egypt's water resources and its total uses. This is what is applied to achieve sustainable development of water resources in Egypt.

Keywords: Environmental Information Systems, Water Resources Management, Sustainable Development 